\title{
Methods in Medicine
}

\section{Hyperbaric oxygen therapy in the battlefield}

\author{
Surg Capt George Verghese (Retd) ${ }^{a}$, Surg Lt Cdr Rohit Verma ${ }^{b, *}$, \\ Surg Lt Cdr Sourabh Bhutani ${ }^{\mathrm{c}}$
}

${ }^{a}$ Former Senior Advisor (Marine Medicine), 4A Pragati's Royale, Facors Layout, Waltair Uplands, Vishakhapatnam 530003, Andhra Pradesh, India

${ }^{\mathrm{b}}$ Graded Specialist (Marine Medicine) \& PMO, Diving School, Naval Base, Kochi 682004, India

${ }^{\mathrm{c}}$ Graded Specialist (Marine Medicine), INS Shankul, c/o Fleet Mail Office, Mumbai 400001, India

\section{A R T I C L E I N F O}

\section{Article history:}

Received 12 November 2011

Accepted 27 April 2012

Available online 30 November 2012

Keywords:

Hyperbaric oxygenation

Battle casualty

Field hospital

Military medicine

\begin{abstract}
A B S T R A C T
Hyperbaric oxygen therapy (HBOT) is increasingly being used in a number of areas of medical practice. It is an accepted adjunctive therapy in conditions such as burns, crush injuries, head injuries, spinal cord injuries, reconstruction surgeries, gas poisonings, radiation injuries, various anaerobic and aerobic infections that are commonly encountered in combat. It is being evaluated as a potential therapy for a variety of illnesses such as Post Traumatic Stress Disorder (PTSD) and High Altitude Cerebral Oedema (HACO) that are typically encountered in a combat scenario. The latest hyperbaric chambers are lightweight, portable and easy to operate. Provisioning of such chambers in the zonal hospitals can prove to be an invaluable resource in combat casualty care and may result in improved outcomes.
\end{abstract}

(c) 2012, Armed Forces Medical Services (AFMS). All rights reserved.

\section{Introduction}

Hyperbaric oxygen therapy (HBOT) is defined as delivery of $100 \%$ oxygen at pressures greater than 1 Atmospheres Absolute (ATA) while the patient is being pressurised in a chamber. HBOT is increasingly being recognised as an important adjunct in a select group of indications for which there is mounting scientific evidence. Till now, due to the cumbersome delivery mechanisms, high costs and inadequate knowledge among referring physicians, its usage was largely restricted to the Armed Forces. ${ }^{1}$ This trend is changing with setting up of hyperbaric facilities by large hospitals and wound care centres in metropolitan cities. Also, since the spectrum of indications of HBOT closely overlaps those of combat casualties, HBOT has been increasingly used by US \&
NATO forces on the veterans of combat theatres of Afghanistan and Iraq.

\section{Mechanism of action of HBOT}

The mechanism of action of hyperbaric oxygen therapy is twofold: physical and physiological. The physical effect is exerted due to increased dissolution of oxygen in the plasma by virtue of Henry's Law leading to improvement in the cellular oxygen supply by raising the tissue-cellular diffusion gradient. This has found utility in hypoxia in a variety of settings as in regional hypoxia in compromised surgical grafts, osteoradionecrosis, problem wounds and crush injuries as well as in global hypoxia in carbon monoxide and

\footnotetext{
* Corresponding author. Tel.: +919497191324.

E-mail address: rohitcalvin@gmail.com (R. Verma). 
cyanide poisoning. ${ }^{2}$ Among the physiological effects, HBOT combats anaerobic infections due to production of free radicals and direct inhibition of the clostridial alpha toxin. It also indirectly acts against aerobic infection by helping in the "oxidative burst". Further, hyperbaric oxygen produces vasoconstriction through a Nitric Oxide Synthase (NOS) mediated response, hence reducing oedema, helps in the formation of the collagen matrix that assists wound healing and angiogenesis and prevents free radical injury through inhibition of ICAM1 and CD18. ${ }^{2}$ These mechanisms make HBOT to be useful in indications such as mixed infections, clostridial myonecrosis, necrotising soft tissue infections, and refractory osteomyelitis. Further, latest studies are elaborating ever new mechanisms of action of HBOT. It is emerging that HBOT increases the number of stem cells in the human body, upregulate expression of antioxidant, and cytoprotective genes and provides neuroprotection in the setting of cerebral ischaemia through modulation of various molecular markers ${ }^{2}$ such as MMP9, HIF $1 \alpha$, Superoxide Dismutase, Nogo - A and others.

\section{Indications of HBOT in the combat theatre}

HBOT has been used for a variety of indications, a large number of them without any scientific basis. Two major international organisations, Undersea \& Hyperbaric Medical Society (UHMS) in the US and European Committee for Hyperbaric Medicine (ECHM) in the EU, have a constantly updated list of indications based on scientific evidence. Most clinical establishments utilise either of these lists which are illustrated in Table 1. The indications that are of relevance from the perspective of combat casualties ${ }^{3,4}$ are listed in Table 2.

Data from an Armed Forces HBOT centre in a tertiary care hospital showed that traumatic wounds formed a significant number of the total number of patients being delivered HBOT as listed in Fig. $1 .^{5}$

It is important to note that HBOT is only an adjunctive treatment for majority of these indications. Due to the limitations of the chambers, large number of patients cannot be accommodated for delivery of HBOT. Hence, carefully selected patients, meeting laid down criteria, will derive maximum benefit. HBOT can prove to be an important factor in improving the prognosis in these patients in whom the standard care is proving to be inadequate.

\section{Role of HBOT in the combat theatre}

The use of HBO2 therapy for the management of combatrelated trauma has been suggested by a number of authors. ${ }^{1-4,6}$ It is apparent from the range of established indications of HBOT that it would be an important force multiplier for the military surgeon. ${ }^{2,3,6}$ It would enable a significant improvement in the prognosis and survival of those injured in war. However, the primary difficulty with employment of this therapeutic modality is providing ready access of combat casualties to hyperbaric chamber facilities. HBOT works best as an adjunct if delivered within the crucial
Table 1 - Indications of hyperbaric oxygen therapy.

Undersea and Hyperbaric Medical Society (UHMS) and

European Committee for Hyperbaric Medicine (ECHM) are two major international organisation which bring out updated lists of indications based on scientific evidence.

Indications accepted by both UHMS and ECHM

1 Air or gas embolism

2 Carbon monoxide poisoning (whether or not complicated by cyanide poisoning)

3 Crush injuries, compartment syndrome and other acute ischaemias

$4 \quad$ Decompression sickness

5 Enhancement of healing in selected problem wounds

$6 \quad$ Necrotising soft tissue infections

$7 \quad$ Refractory osteomyelitis

8 Delayed radiation injury including soft tissue and bone necrosis

9 Burns

Indications accepted only by UHMS

10 Clostridial myositis and myonecrosis (gas gangrene)

11 Exceptional anaemia

12 Intracranial abscess

13 Skin grafts and flaps

Indications accepted only by ECHM

14 Sudden deafness

15 Ophthalmologic disorders

16 Neuroblastoma stage IV

17 Pneumatosis cystoides intestinalis

18 Post-anoxic encephalopathy

few hours of the injury. ${ }^{6}$ Though, logistical issues complicate deployment of hyperbaric chambers into the combat environment. Indeed, size, safety, mobility and operational constraints render co-location of hyperbaric chambers at Level 1 \& 2 (i.e. RAP \& FSC) echelons impractical; however "monoplace" HBOT chambers have a role in the treatment of battle casualties in the subsequent echelons.

Towards this end, provisioning of HBOT chambers should be done in the Level 3 hospitals and above. It is recommended

Table 2 - The indications of hyperbaric oxygen therapy (HBOT) that are of relevance from the perspective of combat casualties.

Accepted indication of utility in a combat theatre

1 Gas gangrene

2 Crush injuries

3 Acute ischaemias including cold induced injuries

4 Necrotising soft tissue infections

5 Osteomyelitis

6 Radiation injury

7 Reconstruction surgery

8 Burns

9 Anaerobic and mixed infections

10 Post-anoxic encephalopathy

Research indications of utility in a

combat theatre

11 Post Traumatic Stress Disorder (PTSD)

12 Head injury

13 Spinal cord injury

14 High altitude cerebral oedema (HACO)
Acute indication Acute indication Acute indication

Acute indication Sub-acute indication Sub-acute indication Sub-acute indication Acute indication Sub-acute indication Acute indication

Sub-acute indication Acute indication Acute indication Acute indication 


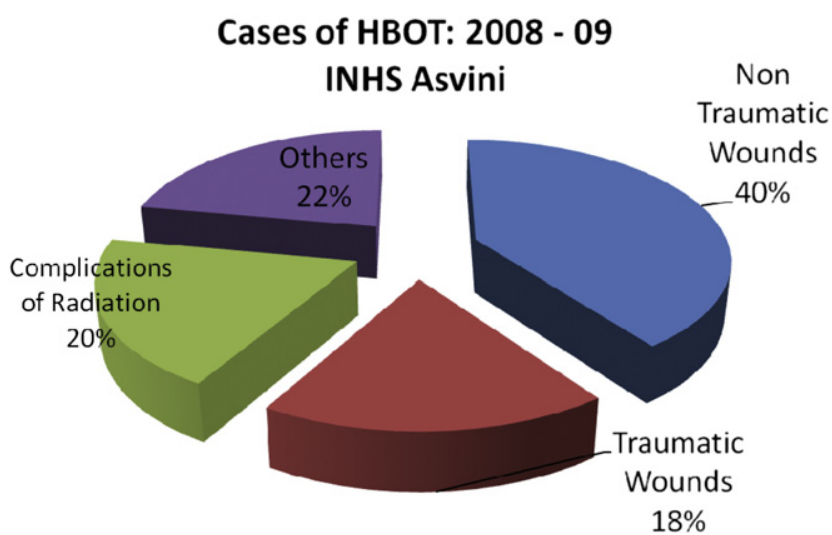

Fig. 1 - Distribution of HBOT cases at a tertiary care centre (adapted from the Poster Presentation ${ }^{5}$ with the kind permission of the author).

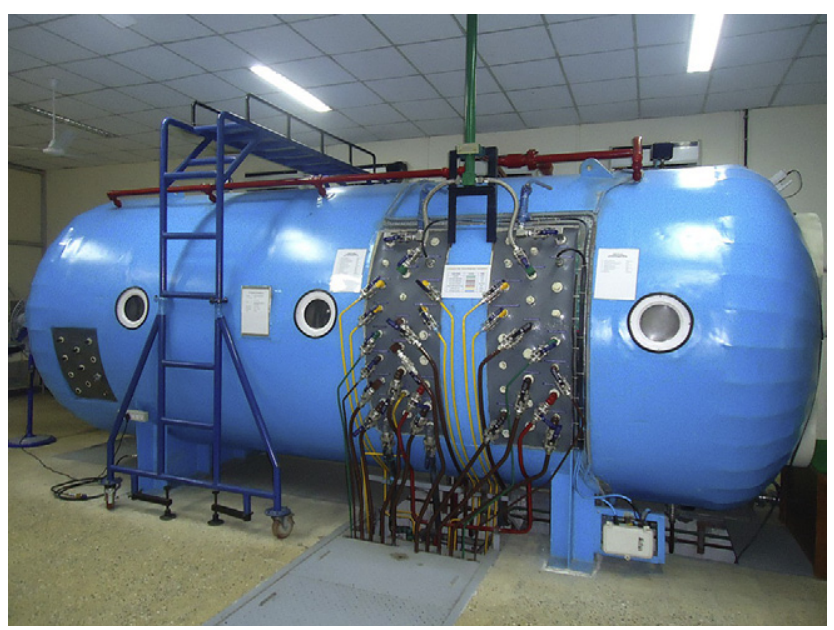

Fig. 2 - An multiplace clinical hyperbaric chamber installed at a command hospital.

that at least two "monoplace" chambers be available at all Level 3 hospitals. There is a requirement for formulating guidelines and prioritisation of patients for optimum utilisation of these chambers. Such chambers in peace time can be a part of the wound care set up of the hospitals and during combat can be used for carefully triaged battle casualties. Training of medical and paramedical personnel in hyperbaric medicine will need to be instituted. Level 4 hospitals (Command Hospitals) can have "multiplace chambers" (Fig. 2) with physicians having specialised training in Hyperbaric Medicine (Marine Medicine or Aviation Medicine specialists) catering to referrals. It is important to lay emphasis on conducting research on the utility of HBOT in various conditions seen in combat as such studies have shown promise elsewhere. Incorporation of hyperbaric oxygen therapy in the battlefield medical set up will go a long way in the comprehensive and holistic management of the combat casualties.

\section{Conclusion}

Combat casualties are treated in an ascending hierarchy of medical echelons in which successive higher levels have greater resources than the lower one. A patient moves along this chain of evacuation as per the triage process. Consequently, the definitive treatment of a combat casualty is a long drawn out process, characterised by a carefully planned and choreographed staged continuum of care. Also, the management of combat casualties is often ridden with delays due to evacuation limitations. These factors often complicate the injuries and require aggressive management as well as unconventional therapies. HBOT can be a useful adjunct in this armamentarium available to the combat physicians and may tilt the balance towards recovery. ${ }^{2,4,6}$

\section{Conflicts of interest}

All authors have none to declare.

\section{REFERENCES}

1. Cramer FS. Care of the injured soldier: a medical readiness role for clinical hyperbaric oxygen therapy. Mil Med. 1985;150(7): 372-375.

2. Fitzpatrick DT. Role of HBO therapy in combat casualty care. US Army Med Dept J. 2000 Jul-Sep:4-9.

3. Fitzpatrick DT. Applications of HBO therapy in army medicine. US Army Med Dept J. 1997 Jan-Feb:17-22.

4. Wright JK. Relevance of hyperbaric oxygen to combat medicine. In: Proceedings of the NATO Research and Technology Office (Human Factors and Medicine) Symposium on Operational Medical Issues in Hypo- and Hyperbaric Conditions 2000 October 16-19. Toronto, Canada. NATO Scientific Report No: RTOMP-062.

5. Bhutani S. Hyperbaric Oxygen Therapy, Poster Presentation at: XXIV Annual Conference of the Marine Medical Society 2009 Oct 24-25. Mumbai, Marine Medical Society.

6. Hart BB. Hyperbaric oxygen therapy - an adjunct to optimal combat trauma management. In: Proceedings of the NATO Research and Technology Office (Human Factors and Medicine) Symposium on Combat Casualty Care in Ground Based Tactical Situations: Trauma Technology and Emergency Medical Procedures 2004 August 16-18. St Peach Beach, USA. NATO Scientific Report No: RTO-MP-HFM-109. 